\title{
Differences in Dihydrotetrabenazine Isomer Concentrations Following Administration of Tetrabenazine and Valbenazine
}

\author{
Heather Skor ${ }^{1}$ (1) Evan B. Smith ${ }^{1} \cdot$ Gordon Loewen $^{1} \cdot$ Christopher F. O'Brien $^{1}$ • \\ Dimitri E. Grigoriadis ${ }^{1} \cdot$ Haig Bozigian ${ }^{1}$
}

Published online: 3 August 2017

(C) The Author(s) 2017. This article is an open access publication

\begin{abstract}
Background Tetrabenazine (TBZ) activity is thought to result from four isomeric dihydrotetrabenazine (HTBZ) metabolites $([+]-\alpha-H T B Z,[-]-\alpha-H T B Z, \quad[+]-\beta-H T B Z$, $[-]-\beta$-HTBZ). Each isomer has a unique profile of vesicular monoamine transporter 2 (VMAT2) inhibition and offtarget binding. Previously published data only report total isomer $(\alpha)$ and $(\beta)$ concentrations. We developed a method to quantify the individual HTBZ isomers in samples from patients with Huntington's disease receiving TBZ. For comparison, concentrations of $[+]-\alpha-H T B Z$, the single active metabolite shared by valbenazine (VBZ) and TBZ, were determined in samples from patients with tardive dyskinesia receiving VBZ.

Methods A liquid chromatography-tandem mass spectrometry (LC-MS/MS) method was developed and validated for quantitation of the four individual HTBZ isomers. Concentrations were determined in serum from patients with Huntington's disease administered TBZ and plasma from patients with tardive dyskinesia administered VBZ once daily.

Results In patients administered TBZ, [-]- $\alpha-\mathrm{HTBZ}$ and $[+]-\beta-H T B Z$ were the most abundant HTBZ isomers while $[-]-\beta-H T B Z$ and $[+]-\alpha-$ HTBZ were present as minor metabolites. Only $[+]-\alpha-$ HTBZ was observed in patients administered VBZ.

Conclusions Based on relative abundance and potency, [+]- $\beta$-HTBZ appears to be the primary contributor to VMAT2 inhibition by TBZ, a finding in contrast with the
\end{abstract}

Heather Skor

hskor@neurocrine.com

1 Neurocrine Biosciences, Inc., 12780 El Camino Real, San Diego, CA 92130, USA generally held assertion that $[+]-\alpha-H T B Z$ is the major contributor. [-]- $\alpha$-HTBZ, the other abundant TBZ metabolite, has much lower VMAT2 inhibitory potency than $[+]-\beta-H T B Z$, but increased affinity for other CNS targets, which may contribute to off-target effects of TBZ. In contrast, pharmacological activity for VBZ is derived primarily from $[+]-\alpha-H T B Z$. Individual HTBZ isomer concentrations provide a more clinically relevant endpoint for assessing on- and off-target effects of TBZ than total isomer concentrations.

\section{Key Points}

This study presents the first reported method for quantifying the four different isomeric dihydrotetrabenazine (HTBZ) metabolites of tetrabenazine, a vesicular monoamine transporter 2 (VMAT2) inhibitor, in serum or plasma samples.

For tetrabenazine, $[-]-\alpha-$ HTBZ and $[+]-\beta-H T B Z$ were the most abundant HTBZ isomers in circulation; for valbenazine, the only observed isomer was $[+]-\alpha-$ HTBZ.

Quantitation of the individual isomers is essential to enable an informed assessment of the risk-to-benefit profile of TBZ under differing clinical conditions.

\section{Introduction}

Modulation of dopamine pathways via inhibition of vesicular monoamine transporter type 2 (VMAT2) is clinically relevant for several neurologic conditions, 
including Huntington's disease, tardive dyskinesia, and Tourette syndrome [1]. Understanding of the pharmacology of VMAT2 inhibition first emerged from early work with reserpine in the 1940 s and then subsequently with discovery of tetrabenazine (TBZ) in the 1950s [2]. Reserpine was found to be a non-selective covalent inhibitor of both VMAT1 and VMAT2 and was associated with reduced monoamine release centrally and peripherally. This combination of effects is associated with hypotension (associated with reduced norepinephrine) and multiple adverse effects in the CNS due to extensive neuronal monoamine depletion [3]. TBZ provided the desirable selectivity for VMAT2 over VMAT1, and avoided severe hypotension, but TBZ was associated with a clinical risk of depression, parkinsonism, sedation, and akathisia [4], some of which may, in part, be due to off-target (non-VMAT2) effects of TBZ and/or its metabolites.

A variety of studies have demonstrated that reserpine and TBZ bind at different sites on VMAT2 [5]. Reserpine causes prolonged and extensive inhibition of pre-synaptic monoamine release due to its nature of covalent binding to VMAT2. In contrast, TBZ is a non-covalent reversible inhibitor of VMAT2 [6]. TBZ is administered as a racemic mixture of two stereoisomers. Racemic TBZ itself is not directly responsible for the pharmacologic effects, but rather, its pharmacologic activity is a consequence of active metabolites of TBZ [7, 8]. Originally these metabolites were described in toto as dihydrotetrabenazine (HTBZ). It was subsequently reported that there were four HTBZ stereoisomers that arise from the two chiral centers in racemic TBZ and from the additional chiral center introduced by reduction of the ketone moiety of TBZ by carbonyl reductase to form the secondary alcohol HTBZ stereoisomers: [+]- $\alpha$-HTBZ, also called (2R,3R,11bR)HTBZ; [-]- $\alpha$-HTBZ or (2S,3S,11bS)-HTBZ; [+]- $\beta$-HTBZ or $\quad(2 \mathrm{~S}, 3 \mathrm{R}, 1 \mathrm{bR})-\mathrm{HTBZ}$; and $[-]-\beta-\mathrm{HTBZ}$ or (2R,3S,11bS)-HTBZ (Fig. 1). Although these metabolites have been synthesized and purified to assess the pharmacological activity of the individual stereoisomers [7, 9], no analytical methods have been developed to date capable of quantifying the circulating levels of the individual HTBZ stereoisomers following TBZ administration. In fact, current published data for TBZ, and its deuterated analog (deutetrabenazine), report only the combined concentrations of enantiomeric pairs of metabolites, historically referred to as $\alpha$-HTBZ (comprising both $[+]-\alpha-H T B Z$ and $[-]-\alpha-H T B Z$ ) and $\beta$-HTBZ (comprising both $[+]-\beta-$ HTBZ, and [-]- $\beta$-HTBZ).

It had previously been assumed that administration of TBZ likely produced inhibition of VMAT2 primarily via the $\alpha$ HTBZ isomers [10-12], with no indication of the relative contributions of each $\alpha$-HBTZ enantiomer, no rationale for assuming the $\beta$-HTBZ isomers did not significantly contribute to VMAT2 inhibition, and very little discussion of the 'offtarget' binding of each of the four individual isomers at other transporters or receptors that could be clinically relevant. Very recent data indicate that these four metabolites indeed have important differences in their pharmacologic profile that may contribute to safety and efficacy of TBZ or other VMAT2 inhibitors [7]. Specifically, the off-target pharmacology of some isomers confirms antagonist activity at dopamine $\mathrm{D}_{2}$ and serotonergic receptors that may be associated with CNS risks, including tardive dyskinesia [7, 13]. It had previously been proposed that exposure to clinically relevant concentrations of dopamine receptor antagonism was not relevant to TBZ use [14], although there was no data associated with the stereoisomer-specific metabolite concentrations to support this claim.

Valbenazine (VBZ), a selective VMAT2 inhibitor recently approved for the treatment of tardive dyskinesia, forms a single HTBZ isomer ([+]- $\alpha$-HTBZ) which is its active metabolite. As shown in Fig. 1, VBZ is administered as a single isomer which generates $[+]-\alpha-H T B Z$ via hydrolysis, a process that does not generate an additional chiral center. The HTBZ isomer formed by VBZ is one of the four possible HTBZ isomers formed by TBZ. As VBZ and TBZ both form HTBZ isomers as active VMAT2 inhibitory metabolites and are in clinical use for movement
Fig. 1 Formation of dihydrotetrabenazine (HTBZ) from tetrabenazine and valbenazine

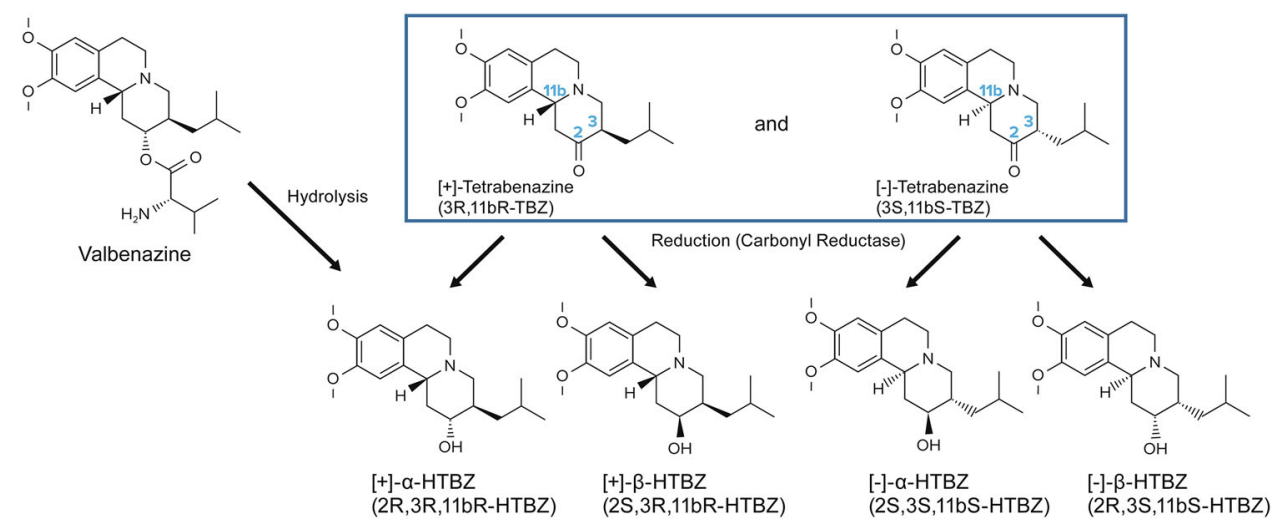


disorders, a comparison of the exposures of HTBZ isomers formed by these two drugs is of interest.

In the current study, to quantify the levels of each independent HTBZ isomer from TBZ, we report the development of a novel liquid chromatography-tandem mass spectrometry (LC-MS/MS) method capable of quantifying the four HTBZ isomers from human serum/plasma at clinically relevant concentrations. This methodology was then used to assess the concentrations of the individual HTBZ isomers in serum samples collected from patients with Huntington's disease following their normal course of treatment with TBZ. Absolute isomer concentrations and relative abundances following TBZ administration were contrasted with isomer concentrations observed in plasma samples collected from patients with tardive dyskinesia who were administered the selective VMAT2 inhibitor VBZ once daily. The potential for each isomer to contribute to on- and off-target activity was assessed based on individual isomer concentration values and reported transporter and receptor binding profiles.

\section{Materials and Methods}

\subsection{Participants}

Serum samples from three patients with Huntington's disease reported to be taking either TBZ 12.5 or $25 \mathrm{mg}$ (Table 1) were purchased from a commercial specimen bank (BioReclamations IVT, Hicksville, NY, USA). All donors provided consent using an Institutional Review Board (IRB)-approved consent form.

Plasma samples were obtained from patients with tardive dyskinesia who received VBZ at doses of either 40 or $80 \mathrm{mg}$ once daily for 48 weeks during a long-term openlabel study [15]. Samples were collected between 2 and
$10 \mathrm{~h}$ after dose administration. For the purposes of this report, equal volumes of plasma samples from five patients per dose level were combined to generate 40 and $80-\mathrm{mg}$ pooled samples. Samples from different subjects were pooled due to limited sample availability.

\subsection{Bioanalytical Method}

Serum (TBZ) and plasma (VBZ) samples and matrixmatched standards and quality controls (QCs) were analyzed using an LC-MS/MS method with pre-column chiral derivatization. Briefly, isotopically labeled $\left(3 \times{ }^{13} \mathrm{C}\right)$ internal standards of all four HTBZ isomers were added to $50 \mu \mathrm{L}$ serum/plasma samples, matrix standards, and/or QC samples. Protein precipitation and phospholipid removal was performed using an Ostro protein precipitation plate (Waters, Millford, MA, USA), 96-well format, with 25-mg sorbent per well. Extracts were dried under nitrogen at $40{ }^{\circ} \mathrm{C}$ and derivatized at ambient temperature in sealed vials for at least $16 \mathrm{~h}$ with constant agitation following the addition of $125 \mu \mathrm{L}$ of (1S)-(-)-camphanic acid chloride at $50 \mathrm{mg} / \mathrm{mL}$ in acetonitrile and $25 \mu \mathrm{L}$ pyridine. After quenching by addition of $30 \mu \mathrm{L}$ of water, solid phase extraction (SPE) was performed to remove excess derivatizing reagents using an Oasis HLB Prime (Waters, Millford, MA, USA) 96-well plate with $30 \mathrm{mg}$ of sorbent per well. The SPE plate was pre-conditioned in multiple steps as follows: $1 \mathrm{~mL}$ of $5 \%$ methanol/95\% water; $2 \times 1 \mathrm{~mL}$ $10 \mathrm{mM}$ ammonium bicarbonate $\mathrm{pH} 8.5 ; 2 \times 1 \mathrm{~mL}$ water; $1 \mathrm{~mL}$ methanol; and $2 \times 1 \mathrm{~mL} 10 \mathrm{mM}$ ammonium bicarbonate $\mathrm{pH}$ 8.5. Quenched samples were added to $1320 \mu \mathrm{L}$ of $10 \mathrm{mM}$ ammonium bicarbonate $\mathrm{pH} 8.5$, mixed, and then loaded into the pre-conditioned SPE plate. The SPE plate was washed with $1 \mathrm{~mL}$ of $5 \%$ methanol/95\% water, then eluted with $2 \times 0.5 \mathrm{~mL}$ of $10 \%$ methanol/90\% acetonitrile with $1 \%$ formic acid. The eluent was dried under nitrogen
Table 1 Characteristics of Huntington's patients taking tetrabenazine

\begin{tabular}{llll}
\hline Parameter & Subject 1 & Subject 2 & Subject 3 \\
\hline Gender & Male & Female & Female \\
Age, years & 50 & 40 & 63 \\
Race & Caucasian & Caucasian & Caucasian \\
Co-medications & Oxcarbazepine $300 \mathrm{mg}$ & Sertraline 100 mg & Donepezil 10 mg \\
& Simvastatin $10 \mathrm{mg}$ & Clonazepam 2 mg & Bupropion 150 mg \\
& Docusate $100 \mathrm{mg}$ & & Clonazepam 0.5 mg \\
& Lorazepam $0.5 \mathrm{mg}$ & & Citalopram 40 mg \\
& Vitamin D3 & & \\
& Aspirin $81 \mathrm{mg}$ & & \\
Tetrabenazine dose, $\mathrm{mg}$ & Bupropion $150 \mathrm{mg}$ & & 12.5 \\
\hline
\end{tabular}


at $40{ }^{\circ} \mathrm{C}$ for approximately $1 \mathrm{~h}$ or until completely dry. Extracts were reconstituted in $300 \mu \mathrm{L}$ of $10 \%$ acetonitrile/ 90\% water for LC-MS/MS analysis.

LC-MS/MS analysis was performed on an instrument comprising a CTC PAL Autosampler (Leap Technologies Inc., Carroboro, NC, USA) and an Agilent 1290 Binary LC pump (Agilent, Santa Clara, CA, USA) coupled to a Sciex API 4000 Qtrap mass spectrometer (Sciex, Framingham, MA, USA). Chromatographic separation was performed using an Acquity BEH C18 $2.1 \times 100 \mathrm{~mm}, 1.7 \mu \mathrm{m}$ column (Waters, Millford, MA, USA) using gradient conditions at a total flow rate of $0.6 \mathrm{~mL} / \mathrm{min}$. Mobile phase A consisted of $0.1 \%$ formic acid in water and mobile phase B was $0.1 \%$ formic acid in acetonitrile. The gradient method started with an initial hold at $24 \%$ B for 0.3 min, linearly increased to $31 \% \mathrm{~B}$ over $10 \mathrm{~min}$ with a 0.2 -min hold, followed by a step to $70 \% \mathrm{~B}$ with a 1-min hold, and finally a 5-min re-equilibration time at initial conditions. All analytes and internal standards were analyzed in positive electrospray ionization (ESI) mode using optimized multiple reaction monitoring (MRM) conditions. MRM channels monitored included derivatized HTBZ isomers $(500.1>302.2)$ and derivatized ${ }^{13} \mathrm{C}$-labeled analogs of HTBZ internal standards $(503.2>305.2)$; dwell times of $65 \mathrm{msec}$ were used for all MRM transitions. Optimized mass spectrometer source settings included Turbo Ion Spray (TIS) source temperature, $500{ }^{\circ} \mathrm{C}$; TIS voltage of $5500 \mathrm{~V}$; curtain gas, 25 psi; ion source gas 1 (GS1), 60 psi; ion source gas 2 (GS2), 50 psi. Instrument data acquisition and analysis was performed using Analyst 1.6.2 software (Sciex, Framingham, MA, USA). Quantification was performed using the internal standard method by determining the peak area ratios for the derivatized HTBZ isomer $(500.1>302.2)$ peaks to the corresponding derivatized ${ }^{13} \mathrm{C}$-labeled analogs of HTBZ internal standard (503.2 > 305.2) peaks. HTBZ isomer concentration values were interpolated from the standard curve over a concentration range of $0.244-125 \mathrm{ng} / \mathrm{mL}$.

\subsection{Bioanalytical Method Validation}

The method was validated with respect to selectivity, matrix effects, sensitivity, linearity, accuracy, precision, intra- and inter-day variability, to demonstrate that the method was acceptable for exploratory analysis of HTBZ isomers in human serum and plasma. Individual batches consisted of a single matrix, either serum or plasma, and included blanks (double and control), standard curves, and QCs as shown in Tables 2, 3, 4 and 5. Interference from endogenous matrix components was assessed using blank human serum and plasma from multiple lots.
Matrix interference due to lipemia was evaluated using visually lipemic human serum. TBZ was included as a potential interference in all serum QCs, and VBZ as a potential interference in all plasma QCs. Isotopically labeled analogs $\left(3 \times{ }^{13} \mathrm{C}\right.$-labeled $)$ of each individual HTBZ isomer were used as internal standards to correct for extraction and derivatization efficiency. Batches were considered to be acceptable if the following criteria were met: the standard curve was linear with a coefficient of determination $\left(r^{2}\right)$ value of the regression line $\geq 0.98$; the average relative errors (RE) of each calibration standard used in the regression performed to establish the calibration curve was within $\pm 20 \%$ of its theoretical concentration; and the percent coefficient of variation $(\mathrm{CV})$ at each $\mathrm{QC}$ level was $\leq 20 \%$. For a given batch the lower limit of quantification (LLOQ) was defined as the concentration of the lowest standard used for generation of the calibration curve.

\section{Results}

\subsection{Bioanalytical Method Validation Results}

As shown in Tables 2, 3, 4 and 5, all batches of serum and plasma for method validation and sample analysis met acceptance criteria. No matrix interferences were observed from endogenous matrix components as observed in the representative blank chromatograms shown in Fig. 2, panels $\mathrm{A}$ and $\mathrm{E}$. Based on these results, the method was acceptable for quantification of HTBZ isomers in serum and plasma.

\subsection{Bioanalytical}

Representative extracted ion chromatograms for a serum blank, a serum standard for HTBZ isomers at the sample batch LLOQ of $0.244 \mathrm{ng} / \mathrm{mL}, 125 \mathrm{ng} / \mathrm{mL}$ serum standard, and a serum sample from a patient administered TBZ are shown in Fig. 2 (panels A, B, C, and D, respectively). Representative extracted ion chromatograms for a plasma blank, a plasma standard for HTBZ isomers at the sample batch LLOQ of $0.488 \mathrm{ng} / \mathrm{mL}, 125 \mathrm{ng} / \mathrm{mL}$ plasma standard, and a pooled plasma sample from patients administered VBZ are shown in Fig. 2 (panels E, F, G, and H, respectively). Chromatographic separation of each of the four isomers is illustrated in the standards. All four isomers were quantifiable in the samples from patients administered TBZ, whereas only the $[+]-\alpha-$ HTBZ isomer was quantifiable in the samples from patients administered VBZ. 
Table 2 Calibration precision and accuracy results for HTBZ isomer standards in human serum ${ }^{\mathrm{a}}$

\begin{tabular}{|c|c|c|c|c|c|c|c|c|c|c|}
\hline \multirow[t]{2}{*}{ Analyte } & \multicolumn{10}{|c|}{ Standard } \\
\hline & Std1 & Std2 & Std3 & Std4 & Std5 & Std6 & Std7 & Std8 & Std9 & Std10 \\
\hline Nominal concentration $(\mathrm{ng} / \mathrm{mL})$ & 0.244 & 0.488 & 0.977 & 1.95 & 3.91 & 7.81 & 15.6 & 31.3 & 62.5 & 125 \\
\hline \multicolumn{11}{|l|}{$[+]-\alpha-\mathrm{HTBZ}$} \\
\hline Mean & 0.242 & 0.489 & 0.924 & 2.00 & 3.83 & 7.77 & 16.0 & 32.6 & 62.1 & 122 \\
\hline SD & 0.01 & 0.03 & 0.06 & 0.12 & 0.23 & 0.47 & 0.69 & 2.3 & 3.2 & 3.0 \\
\hline $\mathrm{CV}, \%$ & 2.7 & 5.9 & 6.8 & 6.1 & 6.0 & 6.1 & 4.3 & 7.0 & 5.1 & 2.4 \\
\hline $\mathrm{RE}, \%$ & -0.79 & 0.08 & -5.4 & 2.6 & -2.1 & -0.61 & 2.5 & 4.3 & -0.64 & -2.4 \\
\hline$N$ & 5 & 3 & 4 & 6 & 6 & 6 & 6 & 5 & 6 & 6 \\
\hline \multicolumn{11}{|l|}{$[-]-\alpha-\mathrm{HTBZ}$} \\
\hline Mean & 0.238 & 0.521 & 0.906 & 1.98 & 3.98 & 7.86 & 15.1 & 33.1 & 62.0 & 120 \\
\hline SD & 0.02 & 0.01 & 0.05 & 0.10 & 0.39 & 0.42 & 0.62 & 2.4 & 3.0 & 3.2 \\
\hline $\mathrm{CV}, \%$ & 7.1 & 1.9 & 5.7 & 4.9 & 9.7 & 5.3 & 4.1 & 7.2 & 4.9 & 2.7 \\
\hline RE, \% & -2.7 & 6.6 & -7.3 & 1.4 & 1.8 & 0.65 & -3.3 & 5.8 & -0.83 & -3.9 \\
\hline$N$ & 5 & 3 & 4 & 6 & 6 & 6 & 6 & 6 & 6 & 6 \\
\hline \multicolumn{11}{|l|}{$[+]-\beta-\mathrm{HTBZ}$} \\
\hline Mean & 0.242 & 0.470 & 0.993 & 2.00 & 3.81 & 8.32 & 15.8 & 31.6 & 58.3 & 123 \\
\hline SD & 0.02 & 0.08 & 0.11 & 0.25 & 0.31 & 0.34 & 1.2 & 2.4 & 3.9 & 11 \\
\hline $\mathrm{CV}, \%$ & 7.3 & 18 & 11 & 12 & 8.0 & 4.1 & 7.5 & 7.6 & 6.7 & 8.6 \\
\hline $\mathrm{RE}, \%$ & -1.0 & -3.7 & 1.7 & 2.5 & -2.5 & 6.5 & 1.3 & 1.2 & -6.7 & -1.6 \\
\hline$N$ & 4 & 3 & 4 & 6 & 6 & 6 & 6 & 6 & 6 & 6 \\
\hline \multicolumn{11}{|l|}{$[-]-\beta-\mathrm{HTBZ}$} \\
\hline Mean & 0.245 & 0.467 & 0.920 & 1.98 & 4.10 & 7.38 & 16.2 & 32.5 & 63.2 & 119 \\
\hline SD & 0.01 & 0.06 & 0.07 & 0.18 & 0.40 & 0.48 & 1.2 & 2.8 & 6.3 & 3.6 \\
\hline $\mathrm{CV}, \%$ & 5.5 & 13 & 7.8 & 9.2 & 9.8 & 6.5 & 7.5 & 8.6 & 10 & 3.0 \\
\hline $\mathrm{RE}, \%$ & 0.27 & -4.4 & -5.8 & 1.3 & 5.0 & -5.5 & 3.9 & 3.9 & 1.2 & -5.1 \\
\hline$N$ & 5 & 3 & 4 & 6 & 6 & 6 & 6 & 6 & 6 & 6 \\
\hline
\end{tabular}

$C V$ coefficient of variation (measure of precision), $H T B Z$ dihydrotetrabenazine, $N$ number of replicates, $R E$ relative error (measure of accuracy), $S D$ standard deviation

${ }^{a}$ Includes data from four calibration batches

\subsection{Circulating Concentrations of Dihydrotetrabenazine (HTBZ) Isomers in Patients Taking Tetrabenazine (TBZ) and Valbenazine (VBZ)}

Concentrations of the individual HTBZ isomers determined in serum samples collected from Huntington's patients taking TBZ, and plasma samples collected from patients with tardive dyskinesia taking VBZ once daily are shown in Table 6 and depicted in Fig. 3.

The most abundant HTBZ metabolites following TBZ administration were $[-]-\alpha-$ HTBZ and $[+]-\beta$-HTBZ. $[-]-\beta-$ HTBZ was observed at relatively lower levels following TBZ administration and $[+]-\alpha-H T B Z$ accounted for only a very small fraction of overall metabolite exposure following TBZ administration. In contrast, the only quantifiable HTBZ isomer following VBZ administration was $[+]-\alpha-$ HTBZ. These results indicate that there is a markedly different steady-state exposure profile of HTBZ metabolites following administration of TBZ and VBZ.

\section{Discussion}

We here report the first demonstration of the simultaneous quantification of the circulating concentrations of the four individual HTBZ isomers in samples collected from patients taking TBZ. The total HTBZ isomer concentrations resulting from administration of TBZ were consistent with previously published steady-state data that presented combined concentrations of enantiomeric pairs of HTBZ isomers [16]. Although the exact dosing regimen of the patients with Huntington's disease is unknown, they were receiving TBZ as a treatment for their disorder and the determined concentrations would be expected to be representative of repeat twice-daily administration of TBZ in 
Table 3 Intra-day and inter-day assay precision and accuracy results for quality control samples of HTBZ isomers in human serum

\begin{tabular}{|c|c|c|c|c|c|c|c|c|c|c|c|}
\hline \multirow[t]{2}{*}{ Analyte } & \multirow[t]{2}{*}{ Nominal concentration $(\mathrm{ng} / \mathrm{mL})$} & \multicolumn{5}{|c|}{ Intra-day assay $^{a}$} & \multicolumn{5}{|c|}{ Inter-day assay $^{\mathrm{b}}$} \\
\hline & & Mean & SD & $\mathrm{CV}, \%$ & RE, \% & $N$ & Mean & SD & $\mathrm{CV}, \%$ & $\mathrm{RE}, \%$ & $N$ \\
\hline \multicolumn{12}{|c|}{$[+]-\alpha-\mathrm{HTBZ}$} \\
\hline LQC & 0.750 & 0.683 & 0.03 & 4.4 & -9.0 & 3 & 0.736 & 0.08 & 10 & -1.9 & 15 \\
\hline MQC & 7.50 & 7.19 & 0.21 & 2.9 & -4.2 & 3 & 7.73 & 0.43 & 5.6 & 3.1 & 12 \\
\hline HQC & 75.0 & 71.2 & 0.50 & 0.7 & -5.1 & 3 & 74.7 & 4.0 & 5.3 & -0.42 & 15 \\
\hline LHQC & 75.0 & 72.4 & 2.6 & 3.6 & -3.5 & 3 & 73.3 & 4.7 & 6.4 & -2.3 & 15 \\
\hline \multicolumn{12}{|c|}{$[-]-\alpha-\mathrm{HTBZ}$} \\
\hline LQC & 0.750 & 0.644 & 0.01 & 1.3 & -14.2 & 3 & 0.657 & 0.03 & 3.8 & -12 & 15 \\
\hline MQC & 7.50 & 6.52 & $\mathrm{NC}$ & $\mathrm{NC}$ & -13.1 & 2 & 6.97 & 0.43 & 6.2 & -7.1 & 11 \\
\hline HQC & 75.0 & 67.1 & 5.4 & 8.1 & -10.5 & 3 & 70.0 & 7.1 & 10 & -6.7 & 15 \\
\hline LHQC & 75.0 & 65.7 & $\mathrm{NC}$ & $\mathrm{NC}$ & -12.4 & 2 & 67.7 & 3.9 & 5.7 & -9.7 & 13 \\
\hline \multicolumn{12}{|c|}{$[+]-\beta-H T B Z$} \\
\hline LQC & 0.750 & 0.629 & $\mathrm{NC}$ & $\mathrm{NC}$ & -16.1 & 2 & 0.689 & 0.07 & 11 & -8.1 & 14 \\
\hline MQC & 7.50 & 6.95 & 0.28 & 4.1 & -7.3 & 3 & 7.40 & 0.51 & 6.8 & -1.4 & 12 \\
\hline HQC & 75.0 & 69.5 & 4.8 & 6.9 & -7.4 & 3 & 74.2 & 6.4 & 8.6 & -1.0 & 14 \\
\hline LHQC & 75.0 & 72.8 & 2.0 & 2.7 & -3.0 & 3 & 74.7 & 5.4 & 7.2 & -0.4 & 15 \\
\hline \multicolumn{12}{|c|}{$[-]-\beta-H T B Z$} \\
\hline LQC & 0.750 & 0.625 & 0.02 & 2.7 & -16.7 & 3 & 0.673 & 0.07 & 11 & -10 & 15 \\
\hline MQC & 7.50 & 6.47 & 0.26 & 4.0 & -13.7 & 3 & 6.96 & 0.62 & 8.8 & -7.2 & 12 \\
\hline HQC & 75.0 & 68.8 & 0.36 & 0.5 & -8.3 & 3 & 71.6 & 5.4 & 7.5 & -4.6 & 15 \\
\hline LHQC & 75.0 & 70.4 & 3.8 & 5.4 & -6.2 & 3 & 73.3 & 5.7 & 7.7 & -2.3 & 15 \\
\hline
\end{tabular}

$C V$ coefficient of variation (measure of precision), $H Q C$ high QC concentration, $H T B Z$ dihydrotetrabenazine, $L H Q C$ lipemic high QC concentration, $L Q C$ low QC concentration, $M Q C$ medium QC concentration, $N$ number of replicates, $N C$ not calculated, $Q C$ quality control, $R E$ relative error (measure of accuracy), $S D$ standard deviation

${ }^{\text {a }}$ Includes data from one of four intra-day QC batches

${ }^{\mathrm{b}}$ Includes data from four inter-day QC batches

that population. The analysis of three serum samples from patients receiving TBZ is sufficient to demonstrate utility of the assay methodology, but is not sufficient to define the effects of intrinsic or extrinsic variables on concentrations of the individual HTBZ isomers. Nonetheless, the pattern of $[-]-\alpha-$ HTBZ and $[+]-\beta$-HTBZ being the most abundant isomers was consistent among the patients, suggesting that the profile may be representative. For VBZ, the concentrations of $[+]-\alpha-$ HTBZ determined in the present study were consistent with those determined using non-chiral methodology, which would be expected as VBZ is administered as a single isomer itself, and thus forms a specific single HTBZ isomer. It is acknowledged that the sample pooling process used for the VBZ samples could have resulted in a dilution of other isomers present in any one individual sample. However, considering that the observed [+]- $\alpha$-HTBZ concentration in the $80-\mathrm{mg}$ pool $(47.5 \mathrm{ng} / \mathrm{mL})$ is approximately 100 -fold higher than the LLOQ of other isomers $(0.488 \mathrm{ng} / \mathrm{mL})$, and given that VBZ is administered as a single isomer where hydrolysis can only generate the single observed isomer $([+]-\alpha-$
HTBZ), these data support that only a single HTBZ isomer is generated following VBZ administration.

The large concentration differences observed for the individual HTBZ isomers are consistent with preliminary in vitro experiments performed in our lab that showed stereo-selective reduction of the TBZ enantiomers by microsomal and cytosolic carbonyl reductase, the metabolic process by which HTBZ isomers are formed (data not shown). However other important factors impacting exposures of the individual HTBZ isomers, such as clearance rates, have not yet been investigated. In vivo experiments using rat and mouse models and the same chiral LC-MS/ MS methodology used in the present study also indicate that the stereo-selectivity in HTBZ isomer formation from TBZ is also species-dependent (manuscript in preparation).

As HTBZ isomers are highly permeable and readily cross the blood-brain barrier (BBB) in non-human species such as rats [16, 17], circulating concentrations of these metabolites provide a reasonable surrogate for concentrations in the brain (the proposed site of action). It is acknowledged that determination of full pharmacokinetic 
Table 4 Calibration accuracy results for HTBZ isomer standards in human plasma ${ }^{a}$

\begin{tabular}{|c|c|c|c|c|c|c|c|c|c|c|}
\hline \multirow[t]{2}{*}{ Analyte } & \multicolumn{10}{|c|}{ Standard } \\
\hline & Std1 & Std2 & Std3 & Std4 & Std5 & Std6 & Std7 & Std8 & Std9 & Std10 \\
\hline Nominal concentration $(\mathrm{ng} / \mathrm{mL})$ & 0.244 & 0.488 & 0.977 & 1.95 & 3.91 & 7.81 & 15.6 & 31.3 & 62.5 & 125 \\
\hline \multicolumn{11}{|l|}{$[+]-\alpha-\mathrm{HTBZ}$} \\
\hline Mean & 0.242 & 0.471 & 0.981 & 2.01 & 4.23 & 8.25 & 14.9 & 32.7 & 57.8 & 117 \\
\hline SD & $\mathrm{NC}$ & $\mathrm{NC}$ & $\mathrm{NC}$ & $\mathrm{NC}$ & $\mathrm{NC}$ & $\mathrm{NC}$ & $\mathrm{NC}$ & $\mathrm{NC}$ & $\mathrm{NC}$ & $\mathrm{NC}$ \\
\hline $\mathrm{CV}, \%$ & $\mathrm{NC}$ & $\mathrm{NC}$ & $\mathrm{NC}$ & $\mathrm{NC}$ & $\mathrm{NC}$ & $\mathrm{NC}$ & $\mathrm{NC}$ & $\mathrm{NC}$ & $\mathrm{NC}$ & $\mathrm{NC}$ \\
\hline $\mathrm{RE}, \%$ & -0.88 & -3.5 & 0.40 & 2.9 & 8.2 & 5.5 & -4.6 & 4.5 & -7.6 & -6.4 \\
\hline$N$ & 2 & 2 & 2 & 2 & 2 & 2 & 2 & 2 & 2 & 2 \\
\hline \multicolumn{11}{|l|}{$[-]-\alpha-\mathrm{HTBZ}$} \\
\hline Mean & 0.234 & 0.507 & 1.01 & 1.97 & 4.13 & 7.38 & 16.3 & 33.3 & 58.8 & 114 \\
\hline SD & $\mathrm{NC}$ & $\mathrm{NC}$ & $\mathrm{NC}$ & $\mathrm{NC}$ & $\mathrm{NC}$ & $\mathrm{NC}$ & $\mathrm{NC}$ & $\mathrm{NC}$ & $\mathrm{NC}$ & $\mathrm{NC}$ \\
\hline $\mathrm{CV}, \%$ & $\mathrm{NC}$ & $\mathrm{NC}$ & $\mathrm{NC}$ & $\mathrm{NC}$ & $\mathrm{NC}$ & $\mathrm{NC}$ & $\mathrm{NC}$ & $\mathrm{NC}$ & $\mathrm{NC}$ & $\mathrm{NC}$ \\
\hline RE, \% & -4.4 & 3.7 & 3.4 & 0.61 & 5.7 & -5.5 & 4.0 & 6.4 & -6.0 & -9.2 \\
\hline$N$ & 2 & 2 & 2 & 2 & 2 & 2 & 2 & 2 & 2 & 2 \\
\hline \multicolumn{11}{|l|}{$[+]-\beta-\mathrm{HTBZ}$} \\
\hline Mean & 0.230 & 0.513 & 0.952 & 2.02 & 3.28 & 7.84 & 17.6 & 32.5 & 59.4 & 123 \\
\hline SD & $\mathrm{NC}$ & $\mathrm{NC}$ & $\mathrm{NC}$ & $\mathrm{NC}$ & $\mathrm{NC}$ & $\mathrm{NC}$ & $\mathrm{NC}$ & $\mathrm{NC}$ & $\mathrm{NC}$ & $\mathrm{NC}$ \\
\hline $\mathrm{CV}, \%$ & $\mathrm{NC}$ & $\mathrm{NC}$ & $\mathrm{NC}$ & $\mathrm{NC}$ & $\mathrm{NC}$ & $\mathrm{NC}$ & $\mathrm{NC}$ & $\mathrm{NC}$ & $\mathrm{NC}$ & $\mathrm{NC}$ \\
\hline RE, \% & -5.8 & 5.0 & -2.5 & 3.4 & -16 & 0.35 & 12 & 3.8 & -5.0 & -1.6 \\
\hline$N$ & 1 & 2 & 2 & 2 & 2 & 2 & 2 & 2 & 2 & 2 \\
\hline \multicolumn{11}{|l|}{$[-]-\beta-\mathrm{HTBZ}$} \\
\hline Mean & 0.218 & 0.512 & 1.06 & 1.95 & 4.26 & 7.36 & 15.9 & 31.0 & 60.3 & 114 \\
\hline SD & $\mathrm{NC}$ & $\mathrm{NC}$ & $\mathrm{NC}$ & $\mathrm{NC}$ & $\mathrm{NC}$ & $\mathrm{NC}$ & $\mathrm{NC}$ & $\mathrm{NC}$ & $\mathrm{NC}$ & $\mathrm{NC}$ \\
\hline $\mathrm{CV}, \%$ & $\mathrm{NC}$ & $\mathrm{NC}$ & $\mathrm{NC}$ & $\mathrm{NC}$ & $\mathrm{NC}$ & $\mathrm{NC}$ & $\mathrm{NC}$ & $\mathrm{NC}$ & $\mathrm{NC}$ & $\mathrm{NC}$ \\
\hline $\mathrm{RE}, \%$ & -11 & 4.9 & 8.5 & -0.16 & 8.9 & -5.9 & 1.8 & -0.80 & -3.5 & -8.8 \\
\hline$N$ & 1 & 2 & 2 & 2 & 2 & 2 & 2 & 2 & 2 & 2 \\
\hline
\end{tabular}

$C V$ coefficient of variation (measure of precision), $H T B Z$ dihydrotetrabenazine, $N$ number of replicates, $N C$ not calculated, $R E$ relative error (measure of accuracy), $S D$ standard deviation

${ }^{\text {a }}$ Includes data from two calibration batches

profiles of these isomers would provide a more complete understanding of the overall relative abundance and exposure/effect relationships of these metabolites and this remains a subject of interest. Nonetheless, these analyses provide the relative abundance of HTBZ isomers at the time of sampling following TBZ and VBZ administration.

\subsection{Contribution of HTBZ Isomers to Vesicular Monoamine Transporter 2 (VMAT2) Inhibition by TBZ and VBZ}

The in vitro VMAT2 inhibitory potencies of individual HTBZ isomers have been determined by several investigators $[6,7,9]$. These studies indicated that only two of the four HTBZ isomers $([+]-\alpha-$ HTBZ and $[+]-\beta-H T B Z)$ have appreciable VMAT2 inhibitory activity. Measured $K_{\mathrm{i}}$ values for [+]- $\alpha$-HTBZ ranged from 0.31 to $1.3 \mathrm{ng} / \mathrm{mL}(0.97-4.2 \mathrm{nM})$ while values for [+]- $\beta$ HTBZ ranged from 3.1 to $4.3 \mathrm{ng} / \mathrm{mL} \quad(9.7-13.4 \mathrm{nM})$, demonstrating that both isomers were potent VMAT2 inhibitors with $[+]-\alpha$-HTBZ being slightly (2- to 4-fold) more potent than $[+]-\beta$-isomer. $K_{\mathrm{i}}$ values determined for the relatively inactive HTBZ isomers ranged from $80 \mathrm{ng} / \mathrm{mL}$ to $7.6 \mu \mathrm{g} / \mathrm{mL}$ $(250-23,700 \mathrm{nM})$ for [-]- $\alpha$-HTBZ and 220 to $786 \mathrm{ng} / \mathrm{mL}$ $(690-2460 \mathrm{nM})$ for [-]- $\beta$-HTBZ. The difference in VMAT inhibitory potency of $[+]-\alpha$-HTBZ and $[-]-\alpha$-HTBZ (both $\alpha-$ isomers) is consistent with a PET imaging study in humans where $[+]-\alpha-$ HTBZ demonstrated dense localization and selective binding in brain regions with the highest expression of VMAT2 compared with [-]- $\alpha$-HTBZ [18].

While there is wide (more than 20-fold) inter-individual variability, the ratio of $\alpha$-HTBZ (combined $[+]-\alpha-$ HTBZ and $[-]-\alpha$-HTBZ) to $\beta$-HTBZ (combined $[+]-\beta-H T B Z$ and [-]- $\beta$-HTBZ) is approximately $3-1$ in patients taking TBZ [19]. The generally higher exposures observed for $\alpha$ HTBZ, the slightly higher potency of $[+]-\alpha-H T B Z$ relative to $[+]-\beta-H T B Z$, and the lack of individual HTBZ isomer 
Table 5 Intra- and inter-day assay precision and accuracy results for quality control samples of HTBZ isomers in human plasma

\begin{tabular}{|c|c|c|c|c|c|c|c|c|c|c|c|}
\hline \multirow[t]{2}{*}{ Analyte } & \multirow[t]{2}{*}{ Nominal concentration $(\mathrm{ng} / \mathrm{mL})$} & \multicolumn{5}{|c|}{ Intra-day assay $^{\mathrm{a}}$} & \multicolumn{5}{|c|}{ Inter-day assay ${ }^{\mathrm{b}}$} \\
\hline & & Mean & SD & $\mathrm{CV}, \%$ & RE, \% & $N$ & Mean & SD & $\mathrm{CV}, \%$ & $\mathrm{RE}, \%$ & $N$ \\
\hline \multicolumn{12}{|c|}{$[+]-\alpha-\mathrm{HTBZ}$} \\
\hline LQC & 0.750 & 0.705 & 0.03 & 4.8 & -6.0 & 3 & 0.666 & 0.05 & 7.4 & -11 & 6 \\
\hline MQC & 7.50 & 7.74 & 0.12 & 1.5 & -3.2 & 3 & 7.17 & 0.65 & 9.0 & -4.5 & 6 \\
\hline HQC & 75.0 & 75.9 & 2.1 & 2.7 & -1.2 & 3 & 71.3 & 5.5 & 7.7 & -5.0 & 6 \\
\hline \multicolumn{12}{|c|}{$[-]-\alpha-$ HTBZ } \\
\hline LQC & 0.750 & 0.737 & 0.02 & 2.3 & -1.8 & 3 & 0.68 & 0.07 & 10.7 & -9.6 & 6 \\
\hline MQC & 7.50 & 8.18 & 0.59 & 7.2 & 9.0 & 3 & 7.44 & 0.89 & 11.9 & -0.8 & 6 \\
\hline HQC & 75.0 & 74.4 & 1.7 & 2.3 & -0.84 & 3 & 69.3 & 5.91 & 8.5 & -7.6 & 6 \\
\hline \multicolumn{12}{|c|}{$[+]-\beta-\mathrm{HTBZ}$} \\
\hline LQC & 0.750 & 0.755 & 0.07 & 9.0 & 0.71 & 3 & 0.749 & 0.08 & 11 & -0.09 & 6 \\
\hline MQC & 7.50 & 8.17 & 0.27 & 3.3 & 8.9 & 3 & 7.54 & 0.79 & 11 & 0.51 & 6 \\
\hline HQC & 75.0 & 77.2 & 2.5 & 3.2 & 2.9 & 3 & 73.1 & 5.4 & 7.40 & -2.6 & 6 \\
\hline \multicolumn{11}{|c|}{$[-]-\beta-\mathrm{HTBZ}$} & \\
\hline LQC & 0.750 & 0.670 & 0.02 & 2.3 & -11 & 3 & 0.634 & 0.05 & 7.7 & -16 & 6 \\
\hline MQC & 7.50 & 7.77 & 0.03 & 0.39 & -3.6 & 3 & 7.42 & 0.75 & 10 & -1.1 & 6 \\
\hline HQC & 75.0 & 75.4 & 3.4 & 4.5 & -0.53 & 3 & 71.7 & 5.3 & 7.4 & -4.4 & 6 \\
\hline
\end{tabular}

$C V$ coefficient of variation (measure of precision), $H Q C$ high QC concentration, $H T B Z$ dihydrotetrabenazine, $L Q C$ low $\mathrm{QC}$ concentration, $M Q C$ medium QC concentration, $N$ number of replicates, $Q C$ quality control, $R E$ relative error (measure of accuracy), $S D$ standard deviation

${ }^{a}$ Includes data from one of two intra-day QC batches

${ }^{\mathrm{b}}$ Includes data from two inter-day QC batches

concentration data have contributed to the common perception that $\alpha$-HTBZ (in particular $[+]-\alpha-H T B Z$ ) was the most important contributor to the overall in vivo inhibition of VMAT2 by TBZ. Results from the present study directly measuring the independent isomers call this supposition into question as measured concentrations of $[+]-\beta-\mathrm{HTBZ}$ were on average more than 20-fold higher than those observed for $[+]-\alpha$-HTBZ following TBZ administration. Even when the slightly higher potency of $[+]-\alpha-$ HTBZ versus $[+]-\beta-H T B Z$ is taken into consideration, the important contribution of $[+]-\beta-H T B Z$ for VMAT2 inhibition following TBZ administration is clear. This primary role of $[+]-\beta-H T B Z$ for VMAT2 inhibition following TBZ administration may help explain the need for a frequent (two to three times daily) dosing schedule for TBZ as the half-life of total $\beta$-HTBZ isomer is relatively short $(5 \mathrm{~h})$ [16]. In contrast to TBZ, the only HTBZ isomer formed by VBZ is $[+]-\alpha-H T B Z$. These data strongly suggest that in vivo VMAT2 inhibition by TBZ and VBZ is in fact caused via different active metabolites.

\subsection{Contribution of HTBZ Isomers to Potential Off- Target Effects}

Binding of individual HTBZ isomers to CNS relevant targets other than VMAT2 has also been investigated [7].
While the HTBZ isomers that are potent inhibitors of VMAT2 $([+]-\alpha-$ HTBZ and [+]- $\beta$-HTBZ) are highly selective, the other two isomers $([-]-\alpha-\mathrm{HTBZ}$ and $[-]-\beta-$ HTBZ) show high-affinity binding to other CNS-relevant targets. Inhibitory constants for [-]- $\alpha-\mathrm{HTBZ}$ were 58 and $23 \mathrm{ng} / \mathrm{mL}$ (180 and $71 \mathrm{nM}$ ) for the $\mathrm{D}_{2}$ and $5-\mathrm{HT}_{7}$ receptors, respectively. Inhibitory constants for [-]- $\beta$-HTBZ were 17 and $1.9 \mathrm{ng} / \mathrm{mL}$ (53 and $5.9 \mathrm{nM}$ ) for the $\mathrm{D}_{2}$ and $5-\mathrm{HT}_{7}$ receptors, respectively. Based on the circulating concentrations of these isomers observed in the samples from patients administered TBZ, pharmacological effects on these targets cannot be excluded.

\subsection{Clinical Considerations}

This study provides a practical method for the routine quantification of individual HTBZ isomers in serum or plasma. Determination of the exposure to the specific HTBZ isomers would be highly valuable in clinical practice to better understand the impact of intrinsic and extrinsic variables on the efficacy and safety profile of TBZ, including the potential for CNS adverse effects or QTc prolongation. Like many therapeutic agents, the individual patient risk-to-benefit ratio of TBZ may be altered by intrinsic and extrinsic variables. Intrinsic variables, such as cytochrome P450 (CYP) 2D6 genotype, have 

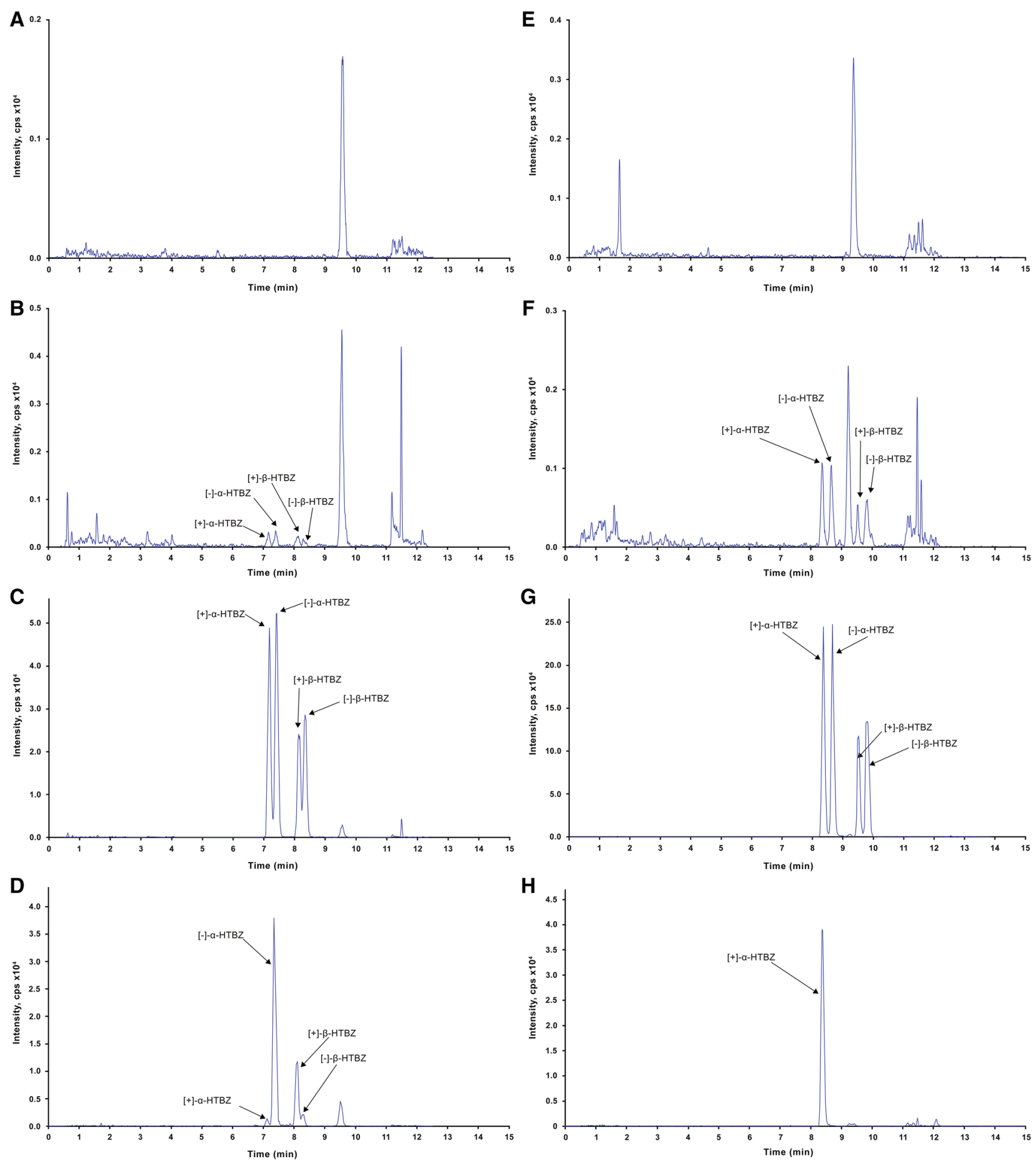

Fig. 2 Representative extracted ion chromatograms for a serum blank (a), a serum standard for HTBZ isomers at the sample batch LLOQ of $0.244 \mathrm{ng} / \mathrm{mL}$ (b), $125 \mathrm{ng} / \mathrm{mL}$ serum standard for HTBZ isomers (c), a representative sample from a patient administered TBZ (d), a representative plasma blank (e), a plasma standard for HTBZ isomers at the sample batch LLOQ of $0.488 \mathrm{ng} / \mathrm{mL}$ (f), a $125-\mathrm{ng} / \mathrm{mL}$ plasma standard for HTBZ isomers (g), and a pooled plasma sample

from patients administered VBZ (h). Serum and plasma assays were performed on different chromatographic column lots that demonstrated differences in column efficiency. When using the more retentive column lot, initial mobile phase composition was adjusted from $24 \%$ B to $26 \%$ B. Hence, slight differences in retention times were observed. $H T B Z$ dihydrotetrabenazine, $L L O Q$ lower limit of quantification, $T B Z$ tetrabenazine, $V B Z$ valbenazine 
Table 6 HTBZ isomer concentrations in serum or plasma following either tetrabenazine or valbenazine administration

\begin{tabular}{|c|c|c|c|c|c|}
\hline \multirow[t]{2}{*}{ Variable } & \multicolumn{3}{|l|}{ Tetrabenazine $^{\mathrm{a}}$} & \multicolumn{2}{|l|}{ Valbenazine $^{\mathrm{b}}$} \\
\hline & Subject 1 & Subject 2 & Subject 3 & Pool A $(40 \mathrm{mg})^{\mathrm{c}}$ & Pool B $(80 \mathrm{mg})^{\mathrm{c}}$ \\
\hline \multicolumn{6}{|l|}{$[+]-\alpha-\mathrm{HTBZ}^{\mathrm{d}}$} \\
\hline Mean \pm SD & $1.13 \pm 0.015$ & $2.08 \pm 0.015$ & $0.512 \pm 0.078$ & $20.1 \pm 1.153$ & $47.5 \pm 0.379$ \\
\hline Replicate 1 & 1.11 & 2.10 & 0.602 & 19.0 & 47.3 \\
\hline Replicate 2 & 1.13 & 2.08 & 0.472 & 21.3 & 47.9 \\
\hline Replicate 3 & 1.14 & 2.07 & 0.461 & 20.0 & 47.2 \\
\hline $\mathrm{CV}, \%$ & 1.4 & 0.7 & 15.3 & 5.7 & 0.8 \\
\hline \multicolumn{6}{|l|}{$[-]-\alpha-\mathrm{HTBZ}^{\mathrm{d}}$} \\
\hline Mean \pm SD & $27.7 \pm 0.907$ & $61.1 \pm 3.460$ & $22.0 \pm 0.100$ & & \\
\hline Replicate 1 & 28.5 & 59.9 & 21.9 & BLQ & BLQ \\
\hline Replicate 2 & 27.8 & 65.0 & 22.0 & BLQ & BLQ \\
\hline Replicate 3 & 26.7 & 58.4 & 22.1 & BLQ & BLQ \\
\hline $\mathrm{CV}, \%$ & 3.3 & 5.7 & 0.5 & & \\
\hline \multicolumn{6}{|l|}{$[+]-\beta-\mathrm{HTBZ}^{\mathrm{d}}$} \\
\hline Mean $\pm \mathrm{SD}$ & $25.1 \pm 0.173$ & $33.4 \pm 1.721$ & $13.3 \pm 0.700$ & & \\
\hline Replicate 1 & 25.3 & 31.4 & 12.5 & BLQ & BLQ \\
\hline Replicate 2 & 25.0 & 34.6 & 13.8 & BLQ & BLQ \\
\hline Replicate 3 & 25.0 & 34.1 & 13.6 & BLQ & BLQ \\
\hline $\mathrm{CV}, \%$ & 0.7 & 5.2 & 5.3 & & \\
\hline \multicolumn{6}{|l|}{$[-]-\beta-\mathrm{HTBZ}^{\mathrm{d}}$} \\
\hline Mean \pm SD & $3.00 \pm 0.078$ & $4.78 \pm 0.214$ & $2.32 \pm 0.200$ & & \\
\hline Replicate 1 & 3.02 & 5.02 & 2.51 & BLQ & BLQ \\
\hline Replicate 2 & 2.91 & 4.69 & 2.11 & BLQ & BLQ \\
\hline Replicate 3 & 3.06 & 4.62 & 2.33 & BLQ & BLQ \\
\hline $\mathrm{CV}, \%$ & 2.6 & 4.5 & 8.6 & & \\
\hline \multicolumn{6}{|c|}{$\begin{array}{l}B L Q \text { below limit of quantification, } C V \text { coefficient of variation, } H T B Z \text { dihydrotetrabenazine, } S D \text { standard } \\
\text { deviation }\end{array}$} \\
\hline \multicolumn{6}{|c|}{ a Tetrabenazine results are from serum samples expressed as $\mathrm{ng} / \mathrm{mL}$} \\
\hline \multicolumn{6}{|c|}{ b Valbenazine results are from plasma sample pools expressed as $\mathrm{ng} / \mathrm{mL}$} \\
\hline \multicolumn{6}{|c|}{$\begin{array}{l}\text { c } \text { BLQ for valbenazine pools: }[-]-\alpha-\mathrm{HTBZ}<0.244 \mathrm{ng} / \mathrm{mL},[+]-\beta-\mathrm{HTBZ}<0.488 \mathrm{ng} / \mathrm{mL},[-]-\beta-\mathrm{HTBZ} \\
<0.488 \mathrm{ng} / \mathrm{mL} \text {. Mean and CV not calculated for isomers with BLQ concentrations }\end{array}$} \\
\hline
\end{tabular}

already been demonstrated to impact clinical use of TBZ in that, due to potential for decreased clearance of HTBZ isomers, the maximum TBZ dose is restricted in patients who are CYP2D6 poor metabolizers (PM). However, without knowledge of the impact of CYP2D6 PM status on exposure to the individual isomers, the optimal TBZ doses to maintain an acceptable risk-to-benefit exposure cannot be determined. Similarly, extrinsic factors such as co-administration with concomitant CYP2D6 inhibitors have been shown to differentially affect exposure to total $\alpha$ - (3fold increase in overall exposure) and $\beta$-isomers (9-fold increase in overall exposure) of HTBZ [4]. However, since the magnitude of their effect on the individual isomers is unknown, the specific change in the risk-to-benefit ratio when co-administering TBZ with CYP2D6 inhibitors cannot be determined. Similar concerns apply to other intrinsic and extrinsic variables that are known to, or could potentially affect HTBZ exposure, including hepatic impairment, renal impairment, and age effects. Differential effects of CYP2D6 inhibitors on $\alpha$ - and $\beta$-isomers may also be of clinical relevance for deutetrabenazine, the deuterated analog of TBZ. Coadministration of paroxetine, a strong CYP2D6 inhibitor, caused a 1.9- and 6.5-fold increase in overall exposure to the total $\alpha$ - and total $\beta$ isomers of deutetrabenazine [20]. However, paroxetine effects on the individual $\alpha$ - and $\beta$-isomers of deutetrabenazine are unknown.

\section{Conclusion}

In summary, this study demonstrated that quantitation of individual HTBZ isomers in plasma or serum is feasible and that, based on the few current samples, exposure to 


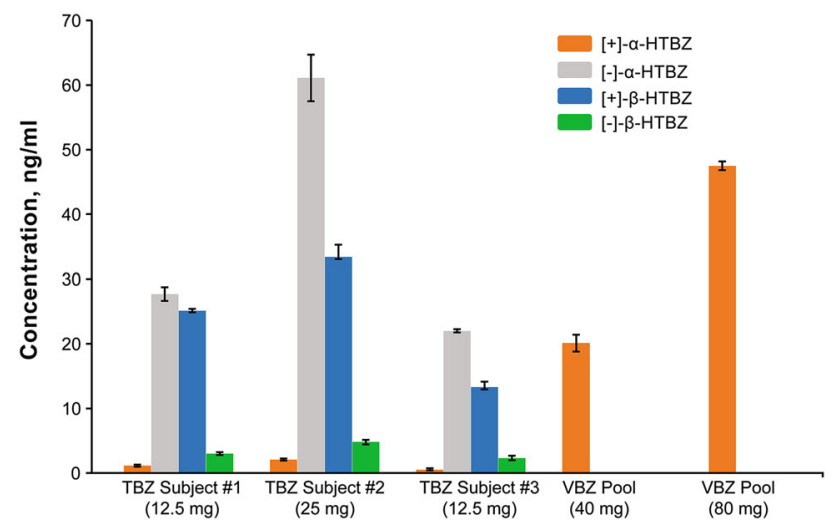

Fig. 3 Concentrations of the individual HTBZ isomers determined in serum samples collected from patients with Huntington's disease taking TBZ and in pooled plasma samples collected from patients with tardive dyskinesia taking VBZ once daily. HTBZ dihydrotetrabenazine, $T B Z$ tetrabenazine, $V B Z$ valbenazine

$[-]-\alpha-$ HTBZ and $[+]-\beta-H$ HBZ may predominate over exposure to $[-]-\beta-$ HTBZ and $[+]-\alpha$-HTBZ. It is important to note that the novel VMAT2 inhibitor, VBZ, produces only the $[+]-\alpha-$ HTBZ isomer, suggesting that the predominant VMAT2 inhibitory activity arises from different circulating metabolites when comparing TBZ with VBZ. Considering the different pharmacologic profiles of the individual HTBZ isomers formed from TBZ, and the potential for individual isomer exposure to be differentially impacted by intrinsic and extrinsic variables, quantitation of the individual isomers should be routine practice during conduct of efficacy, safety, and pharmacokinetic studies of TBZ to enable an informed assessment of the risk-to-benefit profile of TBZ under differing clinical conditions.

Acknowledgements Editorial assistance was provided by Prescott Medical Communications Group (Chicago, Illinois) with support from Neurocrine Biosciences, Inc.

\section{Compliance with Ethical Standards}

This study was supported and conducted by Neurocrine Biosciences, Inc., (San Diego, California). Heather Skor, Evan B. Smith, Gordon Loewen, Christopher F. O'Brien, Dimitri E. Grigoriadis and Haig Bozigian are full-time employees of Neurocrine Biosciences, Inc., with stock or stock options. All plasma and serum samples were obtained from donors (commercial source) or study participants who provided informed and written consent per Institutional Review Board requirements.

Open Access This article is distributed under the terms of the Creative Commons Attribution-NonCommercial 4.0 International License (http://creativecommons.org/licenses/by-nc/4.0/), which permits any noncommercial use, distribution, and reproduction in any medium, provided you give appropriate credit to the original author(s) and the source, provide a link to the Creative Commons license, and indicate if changes were made.

\section{References}

1. Jankovic J. Dopamine depleters in the treatment of hyperkinetic movement disorders. Expert Opin Pharmacother. 2016;17(18):2461-70.

2. Brossi A, Chopard-dit-Jean LH, Schnider O. Syntheseversuche in der emetin-reihe. 3. Mitteilung. 2-hydroxy-hydrobenzo[a]chinolizine. Helv Chim Acta. 1958;41(6):1793-806.

3. Martínez-Olivares R, Villanueva I, Racotta R, Piñón M. Depletion and recovery of catecholamines in several organs of rats treated with reserpine. Auton Neurosci. 2006;128(1-2):64-9.

4. Xenaxine [package insert]. Deerfield, IL: Lundbeck; 2015.

5. Scherman D, Henry JP. Reserpine binding to bovine chromaffin granule membranes. Characterization and comparison with dihydrotetrabenazine binding. Mol Pharmacol. 1984;25(1):113-22.

6. Kilbourn M, Lee L, Vander Borght T, Jewett D, Frey K. Binding of alpha-dihydrotetrabenazine to the vesicular monoamine transporter is stereospecific. Eur $\mathrm{J}$ Pharmacol. 1995;278(3):249-52.

7. Grigoriadis DE, Smith E, Hoare SR, Madan A, Bozigian H. Pharmacologic characterization of valbenazine (NBI-98854) and its metabolites. J Pharmacol Exp Ther. 2017;361:454-61.

8. Jankovic J, Clarence-Smith K. Tetrabenazine for the treatment of chorea and other hyperkinetic movement disorders. Expert Rev Neurother. 2011;11(11):1509-23.

9. Yao Z, Wei X, Wu X, Katz JL, Kopajtic T, Greig NH, et al. Preparation and evaluation of tetrabenazine enantiomers and all eight stereoisomers of dihydrotetrabenazine as VMAT2 inhibitors. Eur J Med Chem. 2011;46(5):1841-8.

10. Kilbourn MR, Lee LC, Heeg MJ, Jewett DM. Absolute configuration of (+)-alpha-dihydrotetrabenazine, an active metabolite of tetrabenazine. Chirality. 1997;9(1):59-62.

11. Acton QA. Huntington's Disease: New Insights for the Healthcare Professional. Atlanta, Georgia: ScholarlyEditions; 2011.

12. Nitoman (tetrabenazine) $25 \mathrm{mg}$ tablets. Irish Medicines Board, summary of product characteristics; April 2014.

13. LeWitt PA. Tardive dyskinesia caused by tetrabenazine. Clin Neuropharmacol. 2013;36(3):92-3.

14. Marshall FJ, Fahn S, Clarence-Smith K. Tetrabenazine as antichorea therapy in Huntington disease: a randomized controlled trial [author reply to correspondence]. Neurology. 2007;68(10):797.

15. Grigoriadis D, Comella C, Remington G, Jimenez R, Burke J, O'Brien C. Efficacy of valbenazine (NBI-98854) in subjects with tardive dyskinesia: results of a long-term extension study (KINECT 3 extension) [abstract]. Neuropsychopharmacol. 2016;41(Suppl S1):S213.

16. U.S. Food and Drug Administration. Xenazine (tetrabenazine) tablets (drug approval package) 2017. https://www.accessdata. fda.gov/drugsatfda_docs/nda/2008/021894s000TOC.cfm.

17. Mehvar R, Jamali F. Concentration-effect relationships of tetrabenazine and dihydrotetrabenazine in the rat. J Pharm Sci. 1987;76(6):461-5.

18. Koeppe RA, Frey KA, Kuhl DE, Kilbourn MR. Assessment of extrastriatal vesicular monoamine transporter binding site density using stereoisomers of [11c]dihydrotetrabenazine. J Cereb Blood Flow Metab. 1999;19(12):1376-84.

19. U.S. Food and Drug Administration. Tetrabenazine briefing document for peripheral and central nervous system advisory committee. 2007. https://www.fda.gov/ohrms/dockets/ac/07/ briefing/2007-4328b1-02-Prestwick.pdf.

20. Austedo [package insert]. North Wales, PA: Teva Pharmaceuticals USA, Inc.; 2017. 